\title{
The Economic Transition Analysis of Resource-dependent Cities
}

\author{
Zenglong Qu \\ University of Massachusetts, Boston, 02125, USA \\ Email: qzenglong@yahoo.com
}

\begin{abstract}
The resource-dependent cities had made great contribution for the economic and social development for the regional economy progress. However, some of them are facing great economic, social and environmental crisis due to the exhaustible resource. The transformation of economy development mode and industrial structural regulation is the solution to the problem. In this paper, the author presents a system dynamics model for these cities in the economy development.
\end{abstract}

Keywords: system dynamics modeling, city development strategy, metropolitan area

\section{Introduction}

In recent years, the urban agglomeration (or: metropolis, megalopolis, UA in the next) is one of hot topics in regional science and geographical economics. Many urban agglomerations in different scales have already emerged or formed in China's different regions since the policy of opening up adoption in 1980s. However, many natural resources, such as coal, oil and other fossil energy are exhaustible and nonrenewable. The more the people dig from the underground, the less they remain. Therefore, it is essential for these cities to do sustainable development researches. In the previous literatures, there are projects that study this problem from the industrial structure adjustment or regulation, or the risk avoidance in the city planning. In this paper, the author will not study it from these perspectives, but from the metropolitan economy or the urban agglomeration (Chincarini and Asherie).

Especially the concept "System Dynamics" (SD) has proved to be advantageous for the dynamic, high-order, nonlinear complicated system (Chincarini and Asherie). The urban agglomeration is one of these systems coupling many subsystems and factors. Thus, the author takes the SD as tools to construct a model to study the development strategy for the mining-dependent or resource-dependent cities.

The paper is arranged as follows. The next section is the system dynamics modeling, it will follow the general SD model steps and develop a high-order SD model. Section Three is the process of the simulation and the method to choose best development strategy for mining-dependent cities in the UAs. Finally, the paper is concluded with a brief summary.

\section{System dynamics solution}

We know that SD model is a structure model which is constructed by state variables and rate variables. The system state can only be changed by rate variables, and the change of rate variable is the result of decision making related to system control policy that is made by the flow control auxiliary variables. The control of social economic system is realized by policy which is imposed on the system from outside and which works through the inside mechanism of the system.

\subsection{System boundary}

The main purpose of developing a SD model of industrial clusters coupling and urban development is to simulate the complicated behaviors of urban economy system, to grasp the behavioral trend of this system and to probe the mechanism of the factors operating in the SD system by the construction of the system structure. The simulation results can be treated as possible track or tendency of the system evolution in the future, so they are valuable for the decision making for the government. The value of variables from the SD model can also offer certain information and quantitative reference for the planning departments ${ }^{[1]}$. In this model, the system boundary is set as the geographical space in the central urban agglomeration in Liaoning province, the economy subsystem, population subsystem, urban natural resource subsystem, environment subsystem. In the city subsystem, it includes mining-dependent cities and other ordinary cities.

\subsection{Subsystems}

As the urban development system is very complicated, it is necessary to divide the system into several subsystems in order to analog the urban development strategy.

\subsubsection{Coupling subsystem}


In the coupling subsystem, there are four subsystems: industries related to the natural resource exploitation, urban that is the main body for the UA, the natural resource that is basic for the mining-dependent cities' development and other cities, and the most important factor people playing the most important role in regional development. The four subsystems do have a coupling relationship, and they influence, correlate and interact each other in the process of the system operating. Figure 1 is basic characteristics of the coupling subsystem.

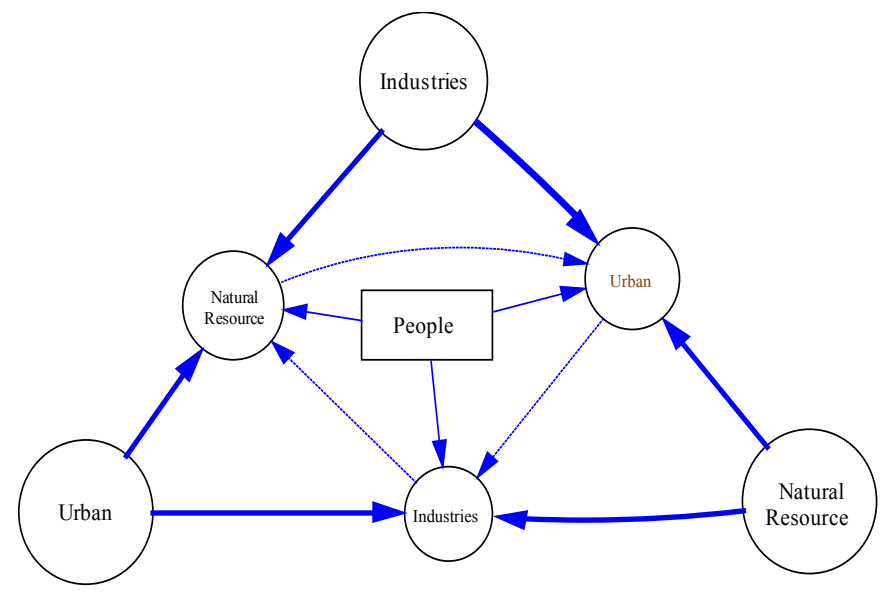

Figure 1. The coupling subsystem of industry development, resource exploration and city extension

\subsubsection{Population subsystem}

In the UA system, the population subsystem includes total population growth, the nonagricultural population growth signifying the scale of city, and the employees in different industries such as the second industry and the tertiary industry. In the urban economics, the nonagricultural population and the percentage in the total population is the index for the urbanization level. In this model, it is also an important index for the UA's development level. The population time series collected from the yearbook of Liaoning province indicated that the nonagricultural population accounted for $22 \% \sim 25 \%$ of total population in the Central Urban Agglomeration in Liaoning province.

\subsubsection{Urban economy subsystem}

The urban economy subsystem is the core of the SD model that includes the added value of three industries, the industrial structure, the employees in each industry, the total industrial value and some other state variables, rate variables, and auxiliaries. Besides, the mining industries value the manufacture industry, and construction industry ${ }^{[2]}$.

\section{Scenarios and simulation}

Dynamic system is time varying, so the decision making in the SD model is a series of decisions. Each decision will make the system transfer from one state to another. The rule that the series of decision making follows is the control policy which is put on the system from outside. So the situation will change after some variables change their value.

\subsection{Parameters calculation}

In the population subsystem, the total population growth rate has a characteristic of "fluctuating in the middle", i.e. it stayed at a high level about $10 \%$ at the first half of the 1990s, and kept at a relatively low level about $1 \sim 3 \%$ after entering the new century. So, the population growth rate in the model is set at about $3 \%$. As mentioned above, the non-agricultural population growth situation is closely related to urbanization process, and this variable in the CUAL is fluctuating at $2 \sim 5 \%$. Thus it can be set at this interval. The other parameters are set using the similar method.

The urban economy subsystem is the core of the model. The industrial structure is very important. The threeindustry structure, employee's structure, value-added structure, output of the equipment manufacture, output value of steel industry, value-added of mining industry, manufacturing industry, construction in the secondary industry, the flow rate variables, auxiliary variables are all consistent with the yearbook in Liaoning province ${ }^{[3]}$.

As for the parameters closely related to other variables, they are determined by linear or nonlinear regression. Take the GDP and urban constructed area(CA) for example, and they have the equation:

$$
\mathrm{CA}=578.6+0.0749 \mathrm{GDP}
$$


$R^{2}=0.9514$

\subsection{Scenarios}

\subsubsection{Model check up}

With Vensim PLE software applied, the reality check is completed. Then with the historical data during 1990-2003 collected and arranged, historical check is also done. From the simulated results, the relative errors range from $-5 . \%$ to $7 \%$, which indicates the SD model is reliable. And to a certain extent it can reveal some complex behaviors of the coupling system.

\subsubsection{Strategy choice}

In order to compare the results and choose the development strategy for the mining-dependent cities, we introduce the concept of coordination judgment function. The coordination function is used to compare two variables in the degree of harmony. It is defined as the index of coordination that one keeps matching relation between every variable in the system in some extent varying the value.

If the time series $X(t), Y(t)$ are keeping pace with each other, i.e. the deviance or distance $C_{v}$ is at minimum, they are in harmony. $C_{v}$ is defined as (3):

$$
\begin{aligned}
C_{v} & =\left[\frac{(X(t)-Y(t))}{\frac{1}{2}(X(t)+Y(t))}\right]^{2} \\
& =4\left[\frac{(X(t)-Y(t))^{2}}{(X(t)+Y(t))^{2}}\right] \\
& =4\left[1-\frac{4 X(t) Y(t)}{(X(t)+Y(t))^{2}}\right]
\end{aligned}
$$
Due to $0 \leq X(t) \leq 1,0 \leq Y(t) \leq 1$, the sufficient and necessary condition for $C_{v}$ minimum is $\frac{4 X(t) Y(t)}{(X(t)+Y(t))^{2}} \rightarrow \max$. So
the coordination function can be written as $(2)$ :

$$
C(t)=[G(t) T(t)]^{\tau}
$$

Where $G(t)=\left[\frac{4 X(t) Y(t)}{(X(t)+Y(t))^{2}}\right]^{\psi}, T(t)=X(t)^{\varphi} Y(t)^{\gamma}, C(t)$ is the harmony degree index, $T(t)$ is the comprehensive index of harmony, $\varphi, \gamma$ is parameter, $\varphi+\gamma=1$.

In this paper, the $X(t)=\left(x_{1 t}, x_{2 t}, \ldots, x_{n t}\right)^{\prime}$ is the development level of the CUAL and the $Y(t)=\left(y_{1 t}, y_{2 t}, \ldots, y_{m t}\right)^{\prime}$ is set of variables of Anshan city. The evaluation index is calculated as (5)(6): 


$$
\begin{gathered}
\hat{X}(t)=\left(\begin{array}{l}
x_{1 t} / \lambda_{1 \max } \\
x_{2 t} / \lambda_{2 \max } \\
\cdots \\
x_{n t} / \lambda_{n \max }
\end{array}\right) \\
\text { or }=\left(\begin{array}{l}
\lambda_{1 \min } / x_{1 t} \\
\lambda_{2 \min } / x_{2 t} \\
\cdots \\
\lambda_{n \min } / x_{n t}
\end{array}\right) \\
\hat{Y}(t)=\left(\begin{array}{l}
y_{1 t} / \lambda_{1 \max } \\
y_{2 t} / \lambda_{2 \max } \\
\cdots \\
y_{m t} / \lambda_{m \max }
\end{array}\right) \\
\text { or }=\left(\begin{array}{l}
\lambda_{1 \min } / y_{1 t} \\
\lambda_{2 \min } / y_{2 t} \\
\cdots \\
\lambda_{m \min } / y_{m t}
\end{array}\right)
\end{gathered}
$$

Where the $\lambda_{i \max }$ and $\lambda_{i \min }$ are the maximum and minimum of the positive index and negative index. When the index is positive, the best is it's maximum value, $\lambda_{i \max }$ is the planning value, standard value or expected value or ideal value of $x_{i t}$, vise via for $y_{i t}$.

\subsubsection{Schemes determination}

According to the simulation and the coordinating function, the scheme for the mining-dependent city in different environments are as follows. When the supposing scene one appears, the urban development strategy is 11; when scene

\begin{tabular}{|c|c|c|c|c|}
\hline & Natural state & Optimistic state & Pessimistic state & Neutral state \\
\hline Developing in natural & Scheme 11 & Scheme12 & Scheme13 & Scheme14 \\
\hline Develop in harmony & Scheme21 & Scheme22 & Scheme23 & Scheme24 \\
\hline Developing in merge & Scheme31 & Scheme 32 & Scheme33 & Scheme34 \\
\hline
\end{tabular}
two appears, it is scheme22; when scene three is true, the best choice is scheme23; when scene four, Anshan's development strategy is scheme 34 . Table. 1 is the scheme plan.

Table 1. The portfolio of industrial development of mining-dependent city

\section{Conclusion}

The urban agglomeration system is a complicated system, which involves many variables. The system dynamics has great advantages in revealing the mechanism. The model of urban development strategy constructed in this paper is consist with the practice of the central urban agglomeration in Liaoning province, and thus the simulation is reliable and can be taken as the policy making reference.

\section{References}

[1] Chincarini, L., Asherie, N. An analytical model for the formation of economic clusters. Regional Science and Urban 
Economics. 2008; 38(3): 252-270.

[2] Koelling, P., Schwandt, M.J. In: Editor (eds.) Health Systems: A Dynamic System-Benefits from system dynamics. 2005; p. 1321-1327.

[3] Portnov, B.A., Pearlmutter, D. Sustainable urban growth in peripheral areas. Progress in Planning. 1999; 52(4): 239. 